\title{
Molecular Characterization of Human Respiratory Adenoviruses Infection in Xining City, China In 2018
}

\author{
Juan $\mathrm{Yu}^{1,3} \cdot$ Shengcang $\mathrm{Zhao}^{3} \cdot$ Huaxiang $\mathrm{Rao}^{2,4}$
}

Received: 18 March 2020 / Accepted: 5 August 2020 / Published online: 14 September 2020

(C) The Author(s) 2020

\section{Dear Editor,}

Adenoviruses are non-enveloped, double-stranded DNA viruses belonging to the Adenoviridae family. To date, 100 unique genotypes of adenoviruses have been identified, from species A to $G$, could cause respiratory tract, gastrointestinal tract and ocular infections in human (Akello et al. 2020). Previous studies have reported that specific adenoviral types are often associated with certain clinical symptoms, epidemiological settings and demographic risk groups. Human adenovirus (HAdV) genotypes HAdV-B, HAdV-C, and HAdV-E are usually associated with respiratory infection; HAdV-A, -D, -F, and -G with gastrointestinal disease; and HAdV-D and $\mathrm{E}$ with ocular diseases in the healthy individuals (Lynch et al. 2011). Although HAdVs infection is mild and self-limited in the healthy individuals, it can be life-threatening in immune-compromised patients (Echavarria 2008).

HAdVs are playing an important role in the respiratory infections, which are responsible for several lower respiratory tract diseases. So far, little data on circulating HAdVs have been collected in Qinghai Province. Our previous study showed that the species HAdV-B3, HAdV-C1 and HAdV-C2 were the prevalent HAdV types in children in Xining City during 2016-2017 (Yu et al. 2019), which was a little different from the strains circulating in other

Huaxiang Rao

raohuaxiang2006006@163.com

1 Department of Basic Medical Sciences, Changzhi Medical College, Changzhi 046000, China

2 Department of Public Health and Preventive Medicine, Changzhi Medical College, Changzhi 046000, China

3 Center of Hygiene Inspection, Qinghai Center for Disease Control and Prevention, Xining 810007, China

4 Institute for Communicable Disease Control and Prevention, Qinghai Center for Disease Control and Prevention, Xining 810007, China provinces of China (Li et al. 1996; Xie et al. 2012; Zou et al. 2012).

The adenovirus capsid is composed of three major proteins: hexon, fiber and penton base. Based on the hypervariable regions, the hexon gene is most commonly used in the adenoviruses classification (Sarantis et al. 2004). Recently, phylogenetic analysis of the fiber gene has also been incorporated to observe the recombination events amongst adenovirus genotypes (Adhikary et al. 2011; Liu et al. 2011). In this study, we performed active surveillance for HAdV infections based on the hexon and fiber gene sequencing in Xining City. This knowledge would benefit for better understanding the prevalence and molecular evolution of HAdVs and might assist with the effective prevention and control of respiratory adenoviruses infection in Xining City.

As we know, from the influenza surveillance data, a large number of influenza-like cases were found negative for influenza virus. Adenoviruses can cause influenza-like illness, so we investigated the adenoviruses prevalence based on influenza surveillance network in our study. Nasopharyngeal swabs from patients with influenza-like illness (fever with body temperature more than $38^{\circ} \mathrm{C}$ accompanied by respiratory symptom such as runny nose, sore throat, and cough) were collected from Qinghai Provincial People's Hospital, Women's and Children's Hospital of Qinghai Province, and Qinghai Red Cross Hospital in 2018.

In this study, a total of 1734 influenza-like cases with influenza virus-negative aged from 1 month to 95 years old were recruited, including 883 males and 851 females, 613 children and 1121 adults. Sampling intervals (from onset to nasopharyngeal swabs collection) were between 1 and 20 days. Then the samples were detected and isolated for HAdVs and performed hexon and fiber gene sequencing according to previous methods (Yu et al. 2019). The results showed that 86 of 1734 influenza-like cases were positive for HAdVs infection in 2018, the positive rate being $4.96 \%$. Among that, positive rate of $4.64 \%$ (41/883) and 
$5.29 \%(45 / 851)$ were detected for males and females respectively, the difference was not statistically significant $\left(\chi^{2}=0.382, P=0.537\right)$. The median age of the positive cases was 9.5 years old, and the positive rate of $15.94 \%$ $(11 / 69)$ was highest in the age of 4 years, followed by $12.00 \%$ in the age of 2 years $(9 / 75)$, and the difference based on age was statistically significant (Fisher's exact probabilities $P<0.001)$. In addition, the median interval time from the incidence of positive cases to sample collection were $2( \pm 2)$ days, with the highest positive rate of $7.91 \%(17 / 215)$ at an interval of 3 days, followed by an interval of 4 days $(6.98 \%)$, and the difference based on sampling intervals had no statistical significance $\left(\chi^{2}=7.407, P=0.192\right.$ ) (Table 1). During 2018, HAdVs infection was observed throughout the year, the positive rate of $8.33 \%$ in July was the highest and $1.71 \%$ in April was the lowest (Fig. 1A).

86 PCR positive samples were inoculated onto Hep-2 cell for virus isolation, and $23 \mathrm{HAdV}$ strains were isolated through 7-21 days post-inoculation, with isolation rate of $26.74 \%$, which was close to that of $21.43 \%$ in the previous study (Thounaojam et al. 2016). The hexon gene sequence has widely been used for the classification of adenovirus types. In this study, the hexon gene of the 23 HAdV isolates was amplified and sequenced using primer designed by Sarantis et al. (Sarantis et al. 2004) (Forward primer: 5' CTGATGTACTACAACAGCACTGGCAACATGGG-3', Reverse primer: 5'-GCGTTGCGGTGGTGGTTAA ATGGGTTTACGTTGTCCAT-3', the amplicon length is 580-605 bp), and the nucleotide sequences were submitted to GenBank (accession numbers MN389388-MN389410). Molecular typing assignments were based on the identity of the closest matching sequences after both BLAST and phylogenetic analysis. Results showed that 9 of the 23 HAdV isolates (8 HAdV-3 and $1 \mathrm{HAdV}-7$ ) belonged to species $\mathrm{B}$ and 12 of the 23 (5 HAdV-1 and 7 HAdV-2) belonged to species HAdV-C, and 2 of the 23 (2 HAdV-4) belonged to species $\mathrm{HAdV}$-E. It revealed that HAdVs genotypes varied in different months and age groups (Fig. 1B, 1C; Table 1). Furthermore, the partial fiber gene was amplified and sequenced using primers designed by $\mathrm{Xu}$ et al. (Xu et al. 2000) (HAdV-B forward primer: 5' TSTACCCYTATGAAGATGAAAGC- $3^{\prime}$, reverse primer: 5'-GGATAAGCTGTAGTRCTKGGCAT-3', the amplicon

Table 1 Characteristics of gender, age and sampling intervals distribution of patients for HAdVs positive in Xining City in 2018.

\begin{tabular}{|c|c|c|c|c|c|c|c|c|c|c|}
\hline \multirow[t]{2}{*}{ Characteristic } & \multirow{2}{*}{$\begin{array}{l}\text { Total } \\
\text { samples (n) }\end{array}$} & \multirow{2}{*}{$\begin{array}{l}\text { HAdV positive } \\
\text { samples (n) }\end{array}$} & \multirow{2}{*}{$\begin{array}{l}\text { Positive } \\
\text { rate }(\%)\end{array}$} & \multicolumn{5}{|c|}{ HAdV isolates (n) } & \multirow{2}{*}{$\begin{array}{l}\chi 2 / \text { Fisher exact } \\
\text { probabilities }\end{array}$} & \multirow[t]{2}{*}{$P$ value } \\
\hline & & & & HAdV-1 & HAdV-2 & HAdV-3 & HAdV-4 & HAdV-7 & & \\
\hline \multicolumn{11}{|l|}{ Gender } \\
\hline Male & 883 & 41 & 4.64 & 0 & 5 & 3 & 2 & 0 & 0.382 & 0.537 \\
\hline Female & 851 & 45 & 5.29 & 5 & 2 & 5 & 0 & 1 & & \\
\hline \multicolumn{11}{|l|}{ Age(year) } \\
\hline$<0.5$ & 47 & 4 & 8.51 & 1 & 0 & 0 & 0 & 0 & - & $<0.001$ \\
\hline $0.5 \sim$ & 69 & 2 & 2.90 & 0 & 0 & 0 & 0 & 0 & & \\
\hline $1 \sim$ & 85 & 9 & 10.59 & 0 & 2 & 0 & 0 & 1 & & \\
\hline $2 \sim$ & 75 & 9 & 12.00 & 1 & 1 & 1 & 0 & 0 & & \\
\hline $3 \sim$ & 59 & 5 & 8.47 & 0 & 0 & 1 & 0 & 0 & & \\
\hline $4 \sim$ & 69 & 11 & 15.94 & 2 & 2 & 2 & 1 & 0 & & \\
\hline $5 \sim$ & 82 & 3 & 3.66 & 0 & 0 & 1 & 1 & 0 & & \\
\hline $10 \sim$ & 39 & 2 & 5.13 & 0 & 0 & 0 & 0 & 0 & & \\
\hline $15 \sim$ & 104 & 5 & 4.81 & 0 & 2 & 0 & 0 & 0 & & \\
\hline $20 \sim$ & 507 & 12 & 2.37 & 1 & 0 & 1 & 0 & 0 & & \\
\hline $40 \sim$ & 367 & 11 & 3.00 & 0 & 0 & 2 & 0 & 0 & & \\
\hline $60 \sim 95$ & 231 & 13 & 5.63 & 0 & 0 & 0 & 0 & 0 & & \\
\hline \multicolumn{11}{|c|}{ Sampling intervals (day) } \\
\hline $0 \sim$ & 230 & 9 & 3.91 & 0 & 1 & 2 & 0 & 0 & 7.407 & 0.192 \\
\hline $1 \sim$ & 579 & 27 & 4.66 & 1 & 4 & 1 & 0 & 0 & & \\
\hline $2 \sim$ & 520 & 25 & 4.81 & 3 & 2 & 1 & 2 & 0 & & \\
\hline $3 \sim$ & 215 & 17 & 7.91 & 1 & 0 & 2 & 0 & 0 & & \\
\hline $4 \sim$ & 86 & 6 & 6.98 & 0 & 0 & 0 & 0 & 1 & & \\
\hline $5 \sim 20$ & 104 & 2 & 1.92 & 0 & 0 & 2 & 0 & 0 & & \\
\hline Total & 1743 & 86 & 4.96 & 5 & 7 & 8 & 2 & 1 & & \\
\hline
\end{tabular}


A

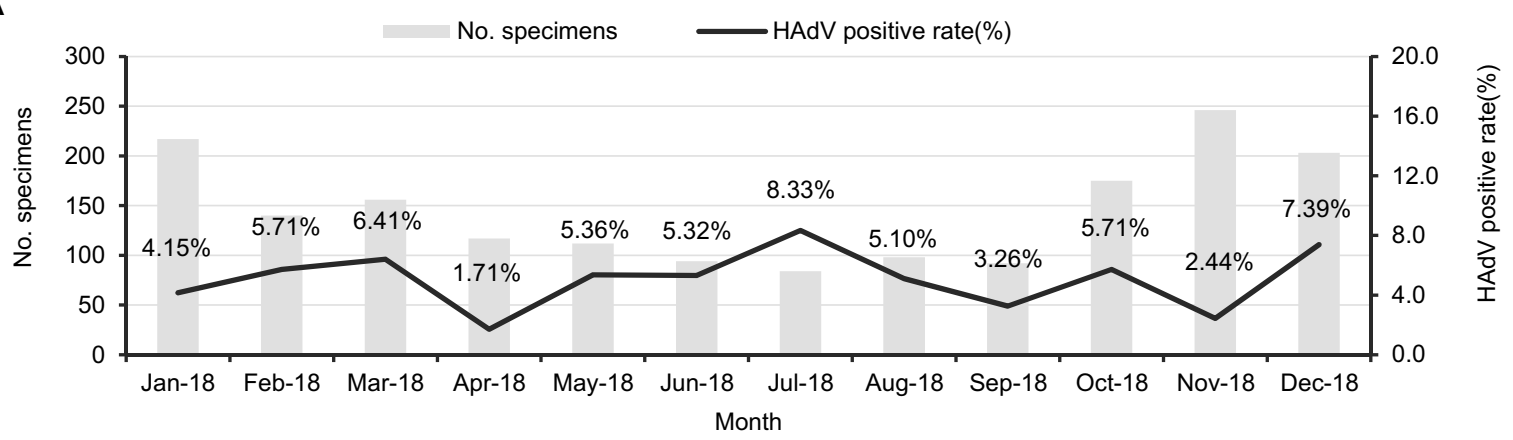

B

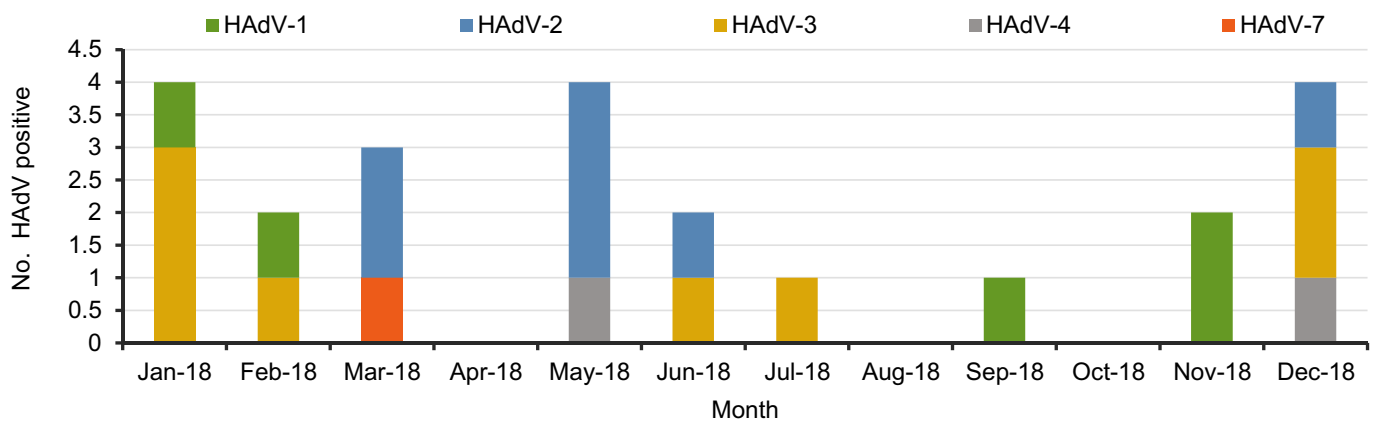

C
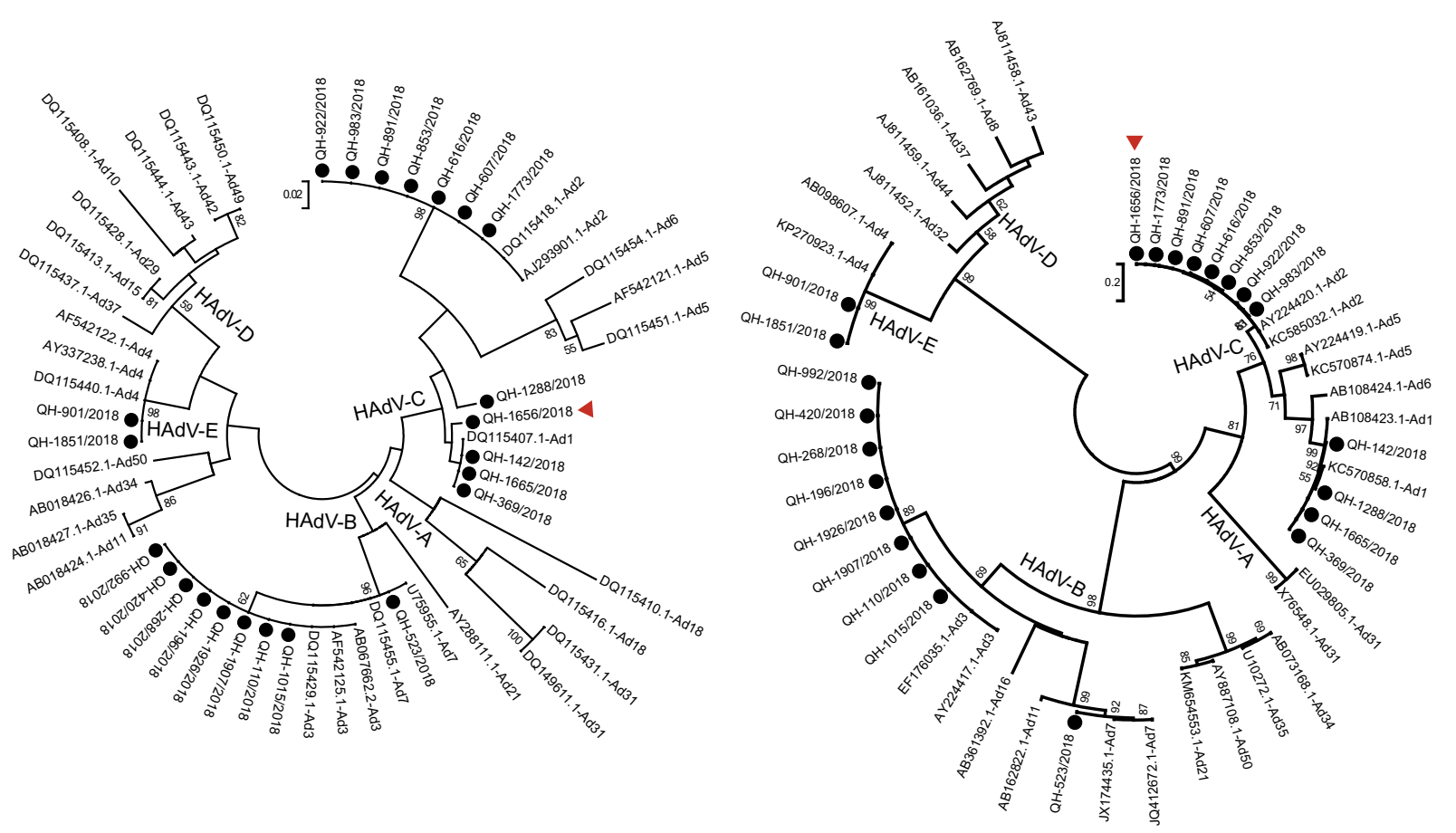

Fig. 1 Prevalence of HAdVs in Xining City in 2018. A Monthly HAdV-positivity in nasopharyngeal specimens in 2018. B Monthly HAdV-strains isolated in 2018. C Phylogenetic tree based on partial hexon (left) and fiber (right) gene sequences of HAdV strains. Black

length is $670-772 \mathrm{bp}$; HAdV-C forward primer: $5^{\prime}$ TATTCAGCATCACCTCCTTTCC- $3^{\prime}$, reverse primer: $5^{\prime}$ AAGCTATGTGGTGGTGGGGC- $3^{\prime}$, the amplicon length is $1988-2000 \mathrm{bp}$; HAdV-E forward primer: $5^{\prime}$ - dots represent Qinghai isolates, and red triangle represents the recombinant strain. Bootstrap proportions (1000 replications) are indicated as a percentage in each node.

TCCCTACGATGCAGACAACG-3', reverse primer: $5^{\prime}$ AGTGCCATCTATGCTATCTCC- $3^{\prime}$, the amplicon length is $967 \mathrm{bp}$ ), and the nucleotide sequences were submitted to GenBank (accession numbers MN389411-MN389433). 
The phylogenetic analysis of the fiber gene showed the same results for the HAdV-B and HAdV-E strains with hexon gene. However, phylogenetic analysis of the fiber gene showed that the results of four HAdV-1 and eight HAdV-2 in HAdV-C cluster were different from that of the hexon gene. It indicated that the recombination events might appear in the Qinghai adenovirus strains (Fig. 1C).

Acute respiratory tract illness is a major health problem globally, and about $5 \%-10 \%$ of acute respiratory infections are caused by adenoviruses (Thounaojam et al. 2016). Previous study showed that species B (HAdV-B3 and HAdV-B7), C (HAdV-C1, HAdV-C2, and HAdV-C5), and E (HAdV-E4) are usually associated with respiratory diseases (Lion 2014). Our study showed that the HAdVs prevalence was $4.96 \%$, and prevalent throughout the year in 2018 in Xining City. Children at the age of 1-4 years were more susceptible to HAdVs infections, which was similar to the previous studies (Esposito et al. 2013; Cheng et al. 2017). HAdV-B3, -B7, HAdV-C1, -C2 and HAdV-E4 were prevalent in Xining City in 2018, among which HAdV-B7 and HAdV-E4 haven't been reported before in this area ( $\mathrm{Yu}$ et al. 2019). And, the most predominant isolates were HAdV-B3, HAdV-C1, and HAdV-C2, which were also the most commonly associated with respiratory HAdVs infection worldwide (Esposito et al. 2016). And the prevalence in 2018 was the same as prevalence during 2016-2017 in Xining City, which indicated that HAdVs infection might be relatively stable.

The identification of adenovirus genotypes is mainly determined by the sequence analysis of the hexon and fiber gene. New adenovirus genotypes are increasingly recognized, and some of the new types may acquire different pathogenicity and cause epidemic outbreaks (Lukashev et al. 2008). For example, HAdV-55, formed due to a recombination between HAdV-11 and HAdV-14 strains, is associated with acute respiratory disease outbreaks (Zhang et al. 2012). In this study, phylogenetic analysis showed one isolate clustered with HAdV-C1 based on hexon gene, but clustered with HAdV-C2 based on fiber gene, which indicated recombination events of HAdVs.

There are some limitations in this study. First, only $26.74 \%$ of all HAdV isolates were obtained and sequenced, which was lower than $56.25 \%$ in 2017 (Yu et al. 2019). Here, HAdVs strains were isolated through 7-21 days postinoculation on Hep-2 cells for three times, but only 23 isolates were obtained, unfortunately no strains isolated in April, August and October. Second, the identification of recombinant strains was incomplete based on hexon and fiber genes, and the complete genome sequencing should be used for identifying the prevalence and the genetic variants of adenoviruses in the future. Third, the clinical characteristics of patients were missed because participants were all from outpatients in this study, as a result, the potential association between the genotypes of HAdVs, the clinical characteristics and the severity of patients could not be analyzed. In addition, HAdV-1, 2, 3, 4, 5, 6, 7 were observed in Xining City during 2 years of surveillance, but no HAdV-55 observed, which might be related to only outpatients being recruited in this study. This suggested that hospitalized patients should be included in further study.

In conclusion, HAdVs surveillance in Qinghai Plateau is limited. In 2018, HAdVs infection was observed in Xining City throughout the year, and HAdV-B3, HAdV-C1 and HAdV-C2 were predominant strains. In addition, one recombinant strain harboring the hexon gene of HAdV-C1 and fiber gene of HAdV-C2 was observed. Our study provided the molecular characteristics of HAdVs in Xining City, which indicated that HAdV recombinant should pay more attention and the corresponding prevention and control strategy should be taken into consideration in this area.

Acknowledgements This study was supported by Doctor Research Startup Foundation of Changzhi Medical College (BS201912, BS201921), Key Project of Qinghai Health and Family Planning Commission (2017-wjzd-08) and Qinghai Thousand People Plan.

\section{Compliance with Ethical Standards}

Conflict of interest The authors declare that they have no conflict of interest.

Animal and Human Rights Statement This study was approved by the Ethics Committee of Qinghai Center for Disease Control and Prevention, the related procedures were performed according to the guideline of National Influenza Surveillance Program (Edition 2017).

Open Access This article is licensed under a Creative Commons Attribution 4.0 International License, which permits use, sharing, adaptation, distribution and reproduction in any medium or format, as long as you give appropriate credit to the original author(s) and the source, provide a link to the Creative Commons licence, and indicate if changes were made. The images or other third party material in this article are included in the article's Creative Commons licence, unless indicated otherwise in a credit line to the material. If material is not included in the article's Creative Commons licence and your intended use is not permitted by statutory regulation or exceeds the permitted use, you will need to obtain permission directly from the copyright holder. To view a copy of this licence, visit http://creativecommons. org/licenses/by/4.0/.

\section{References}

Adhikary AK, Banik U, Okabe N, Fujimoto T (2011) Molecular characterization of human adenovirus type 8 (HAdV-8), including a novel genome type detected in Japan. Jpn J Infect Dis 64:493-498

Akello JO, Kamgang R, Barbani MT, Suter-Riniker F, Leib SL, Ramette A (2020) Epidemiology of human adenoviruses: a 20-year retrospective observational study in hospitalized patients in Bern, Switzerland. Clin Epidemiol 12:353-366 
Cheng JL, Peng CC, Chiu NC, Weng LC, Chiu YY, Chang L, Huang DT, Huang FY, Liu CP, Chi H (2017) Risk factor analysis and molecular epidemiology of respiratory adenovirus infections among children in northern Taiwan, 2009-2013. J Microbiol Immunol Infect 50:418-426

Echavarria M (2008) Adenoviruses in immunocompromised hosts. Clin Microbiol Rev 21:704-715

Esposito S, Daleno C, Prunotto G, Scala A, Tagliabue C, Borzani I, Fossali E, Pelucchi C, Principi N (2013) Impact of viral infections in children with community-acquired pneumonia: results of a study of 17 respiratory viruses. Influenza Other Respir Viruses 7:18-26

Esposito S, Zampiero A, Bianchini S, Mori A, Scala A, Tagliabue C, Sciarrabba CS, Fossali E, Piralla A, Principi N (2016) Epidemiology and clinical characteristics of respiratory infections due to adenovirus in children living in Milan, Italy, during 2013 and 2014. PLoS ONE 11:e0152375

Li QG, Zheng QJ, Liu YH, Wadell G (1996) Molecular epidemiology of adenovirus types 3 and 7 isolated from children with pneumonia in Beijing. J Med Virol 49:170-177

Lion $\mathrm{T}$ (2014) Adenovirus infections in immunocompetent and immunocompromised patients. Clin Microbiol Rev 27:441-462

Liu EB, Ferreyra L, Fischer SL, Pavan JV, Nates SV, Hudson NR, Tirado D, Dyer DW, Chodosh J, Seto D, Jones MS (2011) Genetic analysis of a novel human adenovirus with a serologically unique hexon and a recombinant fiber gene. PLoS ONE 6:e24491

Lukashev AN, Ivanova OE, Eremeeva TP, Iggo RD (2008) Evidence of frequent recombination among human adenoviruses. J Gen Virol 89:380-388
Lynch JP III, Fishbein M, Echavarria M (2011) Adenovirus. Semin Respir Crit Care Med 32:494-511

Sarantis H, Johnson G, Brown M, Petric M, Tellier R (2004) Comprehensive detection and serotyping of human adenoviruses by PCR and sequencing. J Clin Microbiol 42:3963-3969

Thounaojam AD, Balakrishnan A, Mun AB (2016) Detection and molecular typing of human adenoviruses associated with respiratory illnesses in Kerala. Jpn J Infect Dis 69:500-504

Xie L, Yu XF, Sun Z, Yang XH, Huang RJ, Wang J, Yu A, Zheng L, Yu MC, Hu XW, Wang BM, Chen J, Pan JC, Liu SL (2012) Two adenovirus serotype 3 outbreaks associated with febrile respiratory disease and pharyngoconjunctival fever in children under 15 years of age in Hangzhou, China, during 2011. J Clin Microbiol 50:1879-1888

$\mathrm{Xu}$ W, McDonough MC, Erdman DD (2000) Species-specific identification of human adenoviruses by a multiplex PCR assay. J Clin Microbiol 38:4114-4120

Yu J, Li H, Lu NN, Lei YJ, Jiang SY, Zhao SC, Rao HX (2019) Molecular characterization of human respiratory adenovirus infection in children from November 2016 to October 2017 in Xining City, China. Biomed Environ Sci 32:38-41

Zhang Q, Seto D, Cao B, Zhao S, Wan C (2012) Genome sequence of human adenovirus type 55, a re-emergent acute respiratory disease pathogen in China. J Virol 86:12441-12442

Zou L, Zhou J, Li H, Wu J, Mo Y, Chen Q, Fang L, Wu D, Ke C (2012) Human adenovirus infection in children with acute respiratory tract disease in Guangzhou, China. APMIS 120:683-688 